\title{
SUMMATION, TRANSFORMATION, AND EXPANSION FORMULAS FOR BIBASIC SERIES
}

\author{
GEORGE GASPER
}

\begin{abstract}
An indefinite bibasic sum containing three parameters is evaluated and used to derive bibasic extensions of Euler's transformation formula and of a Fields and Wimp expansion formula. It is also used to derive a transformation formula involving four independent bases, a $q$-Lagrange inversion formula, and some quadratic, cubic and quartic summation formulas.
\end{abstract}

\section{INTRODUCTION}

Defining the $q$-shifted factorial by $(a ; q)_{0}=1,(a ; q)_{n}=(1-a)(1-a q)$ $\cdots\left(1-a q^{n-1}\right), n \geq 1$, and letting

$$
\left(a_{1}, a_{2}, \ldots, a_{m} ; q\right)_{n}=\left(a_{1} ; q\right)_{n}\left(a_{2} ; q\right)_{n} \cdots\left(a_{m} ; q\right)_{n},
$$

an $\phi_{s}$ basic hypergeometric series in base $q$ may be defined by

$$
{ }_{r} \phi_{s}\left[\begin{array}{l}
a_{1}, \ldots, a_{r} \\
b_{1}, \ldots, b_{s}
\end{array} q, z\right]=\sum_{n=0}^{\infty} \frac{\left(a_{1}, \ldots, a_{r} ; q\right)_{n}}{\left(q, b_{1}, \ldots, b_{s} ; q\right)_{n}} z^{n}\left[(-1)^{n} q^{\left(\begin{array}{l}
n \\
2
\end{array}\right)}\right]^{1+s-r},
$$

where $\left(\begin{array}{l}n \\ 2\end{array}\right)=n(n-1) / 2$.

Even though there is extensive literature on summation and transformation formulas for these series (see, e.g., Slater [21] and Gasper and Rahman [13]), very little has been published on series containing two or more independent bases. In 1966, Andrews [4] derived a double series expansion for a series containing two independent bases and then used it to derive mock theta function identities, including all of the known identities for the fifth order functions of Ramanujan. One year later, Agarwal and Verma $[1,2]$ used contour integrals and the calculus of residues to derive some transformation formulas expressing certain sums of bibasic series of the form

$$
\sum \frac{\left(a_{1}, \ldots, a_{r} ; q\right)_{n}\left(b_{1}, \ldots, b_{t} ; p\right)_{n}}{\left(c_{1}, \ldots, c_{s} ; q\right)_{n}\left(d_{1}, \ldots, d_{u} ; p\right)_{n}} z^{n}
$$

in terms of other sums of similar series.

Received by the editors October 30, 1987.

1980 Mathematics Subject Classification (1985 Revision). Primary 33A70; Secondary 33A35.

Key words and phrases. Basic hypergeometric series, bibasic expansion formulas, Euler's transformation formula, Lagrange inversion, summation and transformation formulas.

This work was supported in part by the National Science Foundation under grant DMS-8601901. 
In 1973, Carlitz [9, Theorem 5] used the $q$-binomial theorem to prove that the system of equations

$$
\begin{aligned}
f(n)=\sum_{k=0}^{n} \frac{(p ; p)_{n}\left(p^{-k} / a ; q\right)_{n}}{(q ; q)_{n}(p ; p)_{k}(p ; p)_{n-k}}(-1)^{n+k} a^{n} p^{\left(\begin{array}{c}
k+1 \\
2
\end{array}\right)} q^{-\left(\begin{array}{c}
n \\
2
\end{array}\right)} g(k), \\
n=0,1, \ldots, N,
\end{aligned}
$$

is equivalent to the system of equations

$$
\begin{array}{r}
g(n)=\sum_{k=0}^{n} \frac{1-a p^{k} q^{-k}}{1-a p^{n} q^{-k}} \frac{(p ; p)_{n}(q ; q)_{k}}{(p ; p)_{k}(p ; p)_{n-k}\left(p^{-n} / a ; q\right)_{k}} a^{-k} p^{\left(\begin{array}{c}
k \\
2
\end{array}\right)-n k} q^{\left(\begin{array}{c}
k \\
2
\end{array}\right)} f(k), \\
n=0,1, \ldots, N .
\end{array}
$$

Eleven years later, Al-Salam and Verma [3] used the fact that the $n$th $q$ difference of a polynomial of degree less than $n$ is equal to zero to derive the bibasic summation formula

$$
\left(1-\frac{a}{q}\right) \sum_{k=0}^{n} \frac{(-1)^{k}\left(a p^{k} ; q\right)_{n-1}}{(p ; p)_{k}(p ; p)_{n-k}} p^{\left(\frac{n-k}{2}\right)}=\delta_{n, 0}
$$

where $(a ; q)_{-1}=\left(1-a q^{-1}\right)^{-1}$ and $\delta_{n, m}$ is the Kronecker delta function. They used (1.5) to show that Euler's transformation formula

$$
\sum_{n=0}^{\infty} a_{n} b_{n} x^{n}=\sum_{k=0}^{\infty}(-1)^{k} \frac{x^{k}}{k !} f^{(k)}(x) \Delta^{k} a_{0},
$$

where

$$
f(x)=b_{0}+b_{1} x+b_{2} x^{2}+\cdots
$$

and

$$
\Delta^{k} a_{0}=\sum_{j=0}^{k}(-1)^{j}\left(\begin{array}{l}
k \\
j
\end{array}\right) a_{k-j}
$$

and Jackson's [17] $q$-analogue of it have a bibasic extension of the form

$$
\begin{aligned}
\sum_{n=0}^{\infty} A_{n} B_{n}(-x w)^{n}= & \sum_{k=0}^{\infty}(-x)^{k}\left(a q p^{k} ; q\right)_{k-1} \sum_{n=0}^{k} \frac{\left(1-a p^{n} q^{n}\right) w^{n} A_{n}}{(p ; p)_{k-n}\left(a q p^{k} ; q\right)_{n}} \\
& \times \sum_{j=0}^{\infty} \frac{\left(a p^{k} q^{k} ; q\right)_{j}}{(p ; p)_{j}} x^{j} B_{j+k} p^{\left(\begin{array}{l}
3 \\
2
\end{array}\right)}
\end{aligned}
$$

They also observed that (1.7) is equivalent to the fact that the triangular matrix $H=\left(h_{n j}\right)$, where

$$
h_{n j}=\frac{(-1)^{n+j}\left(a q p^{n} ; q\right)_{n-1}\left(1-a p^{j} q^{j}\right)}{(p ; p)_{n-j}\left(a q p^{n} ; q\right)_{j}}
$$


is inverse to the triangular matrix $G=\left(g_{j n}\right)$, where

$$
g_{j n}=\frac{\left(a p^{n} q^{n} ; q\right)_{j-n}}{(p ; p)_{j-n}} p^{\left(\begin{array}{c}
j-n \\
2
\end{array}\right)}
$$

Gessel and Stanton $[15,16]$ pointed out that this is equivalent to the abovementioned Carlitz result and that these are also equivalent to a bibasic analogue of Lagrange inversion for $x /(1-x)^{b+1}$ and for $x /\left(1-x^{r}\right)^{b+1}$. In addition, they used these results to derive identities of the Rogers-Ramanujan type and observed that, since inverse matrices commute, the fact that $G=H^{-1}$ is also equivalent to the bibasic summation formula

$$
\sum_{k=0}^{n} \frac{1-a p^{k} q^{k}}{1-a} \frac{(a ; p)_{k}\left(q^{-n} ; q\right)_{k}}{(q ; q)_{k}\left(a p q^{n} ; p\right)_{k}} q^{n k}=\delta_{n, 0}
$$

Mizan Rahman [19] gave another proof of (1.10) and used the special cases $p=q^{2}$ and $p=q^{3}$ to derive some quadratic and cubic summation formulas. The author observed that Rahman's proof of (1.10) could be extended to infinite series to give

$$
\sum_{k=0}^{\infty} \frac{1-a p^{k} q^{k}}{1-a} \frac{(a ; p)_{k}\left(b^{-1} ; q\right)_{k}}{(q ; q)_{k}(a b p ; p)_{k}} b^{k}=0
$$

when $\max (|p|,|q|,|b|)<1$, and that this formula could in turn be extended to the transformation formulas

$$
\begin{aligned}
\sum_{k=0}^{\infty} & \frac{1-a p^{k} q^{k}}{1-a} \frac{(a ; p)_{k}(c / b ; q)_{k}}{(q ; q)_{k}(a b p ; p)_{k}} b^{k} \\
& =\frac{1-c}{1-b} \sum_{k=0}^{\infty} \frac{(a p ; p)_{k}(c / b ; q)_{k}}{(q ; q)_{k}(a b p ; p)_{k}}(b q)^{k} \\
& =\frac{1-c}{1-a b p} \sum_{k=0}^{\infty} \frac{(a p ; p)_{k}(c q / b ; q)_{k}}{(q ; q)_{k}\left(a b p^{2} ; p\right)_{k}} b^{k} \\
& =\frac{(1-c)(a p ; p)_{\infty}}{(1-b)(a b p ; p)_{\infty}} \sum_{k=0}^{\infty} \frac{(b ; p)_{k}\left(c q p^{k} ; q\right)_{\infty}}{(p ; p)_{k}\left(b q p^{k} ; q\right)_{\infty}}(a p)^{k}
\end{aligned}
$$

when $\max (|p|,|q|,|a p|,|b|)<1$, where

$$
(a ; q)_{\infty}=(1-a)(1-a q)\left(1-a q^{2}\right) \cdots, \quad|q|<1
$$

After receiving a copy of these formulas, William Gosper observed in a letter to the author that the series on the left sides of (1.10) and (1.11) telescope, yielding the indefinite bibasic sum

$$
\sum_{k=0}^{n} \frac{1-a p^{k} q^{k}}{1-a} \frac{(a ; p)_{k}(c ; q)_{k}}{(q ; q)_{k}(a p / c ; p)_{k}} c^{-k}=\frac{(a p ; p)_{n}(c q ; q)_{n}}{(q ; q)_{n}(a p / c ; p)_{n}} c^{-n}
$$

where $n=0,1,2, \ldots$. 
In this paper we shall extend (1.13) to the bibasic indefinite summation formula

$$
\begin{gathered}
\sum_{k=0}^{n} \frac{\left(1-a p^{k} q^{k}\right)\left(1-b p^{k} q^{-k}\right)}{(1-a)(1-b)} \frac{(a, b ; p)_{k}(c, a / b c ; q)_{k}}{(q, a q / b ; q)_{k}(a p / c, b c p ; p)_{k}} q^{k} \\
\quad=\frac{(a p, b p ; p)_{n}(c q, a q / b c ; q)_{n}}{(q, a q / b ; q)_{n}(a p / c, b c p ; p)_{n}}
\end{gathered}
$$

and give several applications of it; including extensions of (1.7)-(1.9), some quadratic, cubic, and quartic summation formulas, a transformation formula for a series containing four independent bases, and extensions of Verma's [25, p. 349] extension of the Fields and Wimp [11] expansion formula

$$
\begin{aligned}
&{ }_{r+t} F_{s+u}\left[\begin{array}{l}
\left.a_{R}, c_{T} ; x w\right]= \\
b_{S}, d_{U}
\end{array} \sum_{n=0}^{\infty} \frac{\left(a_{R}\right)_{n}(\alpha)_{n}(\beta)_{n}}{\left(b_{S}\right)_{n}(\gamma+n)_{n}} \frac{(-x)^{n}}{n !}\right. \\
& \times_{r+2} F_{s+1}\left[\begin{array}{c}
\left.n+\alpha, n+\beta, n+a_{R} ; x\right] \\
1+2 n+\gamma, n+b_{S}
\end{array}\right] \\
& \times{ }_{t+2} F_{u+2}\left[\begin{array}{c}
\left.-n, n+\gamma, c_{T} ; w\right] \\
\alpha, \beta, d_{U}
\end{array}\right]
\end{aligned}
$$

where

$$
(a)_{0}=1, \quad(a)_{n}=a(a+1) \cdots(a+n-1), \quad n \geq 1
$$

is the shifted factorial function and the contracted notations used are $a_{R}$ for $a_{1}, a_{2}, \ldots, a_{r},\left(a_{R}\right)_{n}$ for $\left(a_{1}\right)_{n}\left(a_{2}\right)_{n} \cdots\left(a_{r}\right)_{n}$, and $n+a_{R}$ for $n+a_{1}, n+$ $a_{2}, \ldots, n+a_{r}$.

\section{DERIVATION OF (1.14) AND SOME EXTENSIONS}

Set

$$
s_{n}=\frac{(a p, b p ; p)_{n}(c q, a q / b c ; q)_{n}}{(q, a q / b ; q)_{n}(a p / c, b c p ; p)_{n}}
$$

for $n=0,1,2, \ldots, s_{-1}=0$, and define the difference operator $\Delta$ by

$$
\Delta s_{n}=s_{n}-s_{n-1}
$$

Then $\Delta s_{0}=s_{0}=1$,

$$
\sum_{k=0}^{n} \Delta s_{k}=s_{n}, \quad n \geq 0
$$


and, hence, (1.14) follows from the observation that

$$
\begin{aligned}
\Delta s_{k}= & s_{k}-s_{k-1} \\
= & \frac{(a p, b p ; p)_{k-1}(c q, a q / b c ; q)_{k-1}}{(q, a q / b ; q)_{k}(a p / c, b c p ; p)_{k}} \\
& \quad \times\left\{\left(1-a p^{k}\right)\left(1-b p^{k}\right)\left(1-c q^{k}\right)\left(1-a q^{k} / b c\right)\right. \\
& \left.\quad-\left(1-q^{k}\right)\left(1-a q^{k} / b\right)\left(1-a p^{k} / c\right)\left(1-b c p^{k}\right)\right\} \\
= & \frac{\left(1-a p^{k} q^{k}\right)\left(1-b p^{k} q^{-k}\right)}{(1-a)(1-b)} \frac{(a, b ; p)_{k}(c, a / b c ; q)_{k}}{(q, a q / b ; q)_{k}(a p / c, b c p ; p)_{k}} q^{k}
\end{aligned}
$$

for $k=1,2, \ldots$. Now that $(1.14)$ is known, it can also be verified by induction on $n$.

The series in (1.14) is partially of well-poised type in the sense that $b(a q / b)=$ $a q$ and $c(a p / c)=(a / b c)(b c p)=a p$. Gosper's formula (1.13) follows from (1.14) by letting $b$ tend to zero or infinity. Formula (1.14) also holds for $n=\infty$, provided that $|p|<1$ and $|q|<1$, whereas the right side of (1.13) tends to zero as $n \rightarrow \infty$ if $|c|>1$, yielding formula (1.11) with $b=c^{-1}$.

When $c=q^{-n}, n=0,1, \ldots$, formula (1.14) reduces to

$$
\sum_{k=0}^{n} \frac{\left(1-a p^{k} q^{k}\right)\left(1-b p^{k} q^{-k}\right)}{(1-a)(1-b)} \frac{(a, b ; p)_{k}\left(q^{-n}, a q^{n} / b ; q\right)_{k}}{(q, a q / b ; q)_{k}\left(a p q^{n}, b p q^{-n} ; p\right)_{k}} q^{k}=\delta_{n, 0}
$$

since $\left(q^{1-n} ; q\right)_{n}=0$ for $n \geq 1$. This formula was independently derived in an equivalent form by Bressoud $[8, \S 4]$.

Note that by replacing $a, b, c$ in (1.14) by $p^{a}, p^{b}, p^{c}$, setting $p=q^{\lambda}$ and letting $q \rightarrow 1$, we obtain the limit cases

$$
\begin{gathered}
\sum_{k=0}^{n} \frac{(a+k+k / \lambda)(b+k-k / \lambda)}{a b} \frac{(a)_{k}(b)_{k}(\lambda c)_{k}(\lambda a-\lambda b-\lambda c)_{k}}{k !(\lambda a+1-\lambda b)_{k}(a+1-c)_{k}(b+c+1)_{k}} \\
=\frac{(a+1)_{n}(b+1)_{n}(\lambda c+1)_{n}(\lambda a+1-\lambda b-\lambda c)_{n}}{n !(\lambda a+1-\lambda b)_{n}(a+1-c)_{n}(b+c+1)_{n}}
\end{gathered}
$$

and

$$
\begin{gathered}
{ }_{6} F_{5}\left[\begin{array}{c}
a, 1+\lambda a /(\lambda+1), 1+\lambda b /(\lambda-1), b, \lambda c, \lambda a-\lambda b-\lambda c \\
\lambda a /(\lambda+1), \lambda b /(\lambda-1), \lambda a+1-\lambda b, a+1-c, b+c+1
\end{array} ; 1\right] \\
=\frac{\Gamma(\lambda a+1-\lambda b) \Gamma(a+1-c) \Gamma(b+c+1)}{\Gamma(a+1) \Gamma(b+1) \Gamma(\lambda c+1) \Gamma(\lambda a+1-\lambda b-\lambda c)} .
\end{gathered}
$$

It should also be noted that when $p=q$ formula (1.14) reduces to

$$
\sum_{k=0}^{n} \frac{1-a q^{2 k}}{1-a} \frac{(a, b, c, a / b c ; q)_{k}}{(q, a q / b, a q / c, b c q ; q)_{k}} q^{k}=\frac{(a q, b q, c q, a q / b c ; q)_{n}}{(q, a q / b, a q / c, b c q ; q)_{n}}
$$


which is the special case $e=a q^{1+n}$ of Jackson's sum [21, (IV.8), p. 247]

$$
\begin{aligned}
& { }_{8} \phi_{7}\left[\begin{array}{c}
a, q \sqrt{a},-q \sqrt{a}, b, c, d, e, q^{-n} \\
\left.\sqrt{a},-\sqrt{a}, a q / b, a q / c, a q / d, a q / e, a q^{1+n} ; q, q\right]
\end{array}\right] \\
& =\frac{(a q, a q / b c, a q / c d, a q / b d ; q)_{n}}{(a q / b, a q / c, a q / d, a q / b c d ; q)_{n}},
\end{aligned}
$$

where $a^{2} q=b c d e q^{-n}$. It was in trying to derive a bibasic extension of (1.13) which would reduce to (2.6) when $p=q$ that the author discovered the choice of $s_{n}$ in (2.1). One may, of course, further extend (1.14) by proceeding as above with $s_{n}$ replaced by, for example,

$$
s_{n}=\frac{(a ; p)_{n}(b ; q)_{n}(c ; P)_{n}(d ; Q)_{n}}{\left(e ; p^{\prime}\right)_{n}\left(f ; q^{\prime}\right)_{n}\left(q ; P^{\prime}\right)_{n}\left(h ; Q^{\prime}\right)_{n}},
$$

but then $\Delta s_{k}$ does not simplify as in (2.2), except in special cases.

Formula (1.14) can be extended to a transformation formula containing four independent bases. Just observe that, since

$$
\sum_{k=0}^{n} a_{k} \sum_{j=0}^{n-k} A_{j}=\sum_{k=0}^{n} A_{k} \sum_{j=0}^{n-k} a_{j}
$$

by a change in order of summation, if we take

$$
a_{k}=\frac{\left(1-a p^{k} q^{k}\right)\left(1-b p^{k} q^{-k}\right)}{(1-a)(1-b)} \frac{(a, b ; p)_{k}(c, a / b c ; q)_{k}}{(q, a q / b ; q)_{k}(a p / c, b c p ; p)_{k}} q^{k}
$$

and

$$
A_{k}=\frac{\left(1-A P^{k} Q^{k}\right)\left(1-B P^{k} Q^{-k}\right)}{(1-A)(1-B)} \frac{(A, B ; P)_{k}(C, A / B C ; Q)_{k}}{(Q, A Q / B ; Q)_{k}(A P / C, B C P ; P)_{k}} Q^{k},
$$

then we obtain

$$
\begin{aligned}
\sum_{k=0}^{n} & \frac{\left(1-a p^{k} q^{k}\right)\left(1-b p^{k} q^{-k}\right)}{(1-a)(1-b)} \frac{(a, b ; p)_{k}(c, a / b c ; q)_{k}}{(q, a q / b ; q)_{k}(a p / c, b c p ; p)_{k}} \\
& \times \frac{\left(C P^{-n} / A, P^{-n} / B C ; P\right)_{k}\left(Q^{-n}, B Q^{-n} / A ; Q\right)_{k}}{\left(Q^{-n} / C, B C Q^{-n} / A ; Q\right)_{k}\left(P^{-n} / A, P^{-n} / B ; P\right)_{k}} q^{k} \\
= & \frac{(a p, b p ; p)_{n}(c q, a q / b c ; q)_{n}}{(q, a q / b ; q)_{n}(a p / c, b c p ; p)_{n}} \frac{(Q, A Q / B ; Q)_{n}(A P / C, B C P ; P)_{n}}{(A P, B P ; P)_{n}(C Q, A Q / B C ; Q)_{n}} \\
& \times \sum_{k=0}^{n} \frac{\left(1-A P^{k} Q^{k}\right)\left(1-B P^{k} Q^{-k}\right)}{(1-A)(1-B)} \frac{(A, B ; P)_{k}(C, A / B C ; Q)_{k}}{(Q, A Q / B ; Q)_{k}(A P / C, B C P ; P)_{k}} \\
& \times \frac{\left(c p^{-n} / a, p^{-n} / b c ; p\right)_{k}\left(q^{-n}, b q^{-n} / a ; q\right)_{k}}{\left(q^{-n} / c, b c q^{-n} / a ; q\right)_{k}\left(p^{-n} / a, p^{-n} / b ; p\right)_{k}} Q^{k}
\end{aligned}
$$

for $n=0,1,2, \ldots$, which reduces to $(1.14)$ when $C=1$. 
When $p=P=Q=q,(2.10)$ reduces to a transformation formula for "split-poised" ${ }_{10} \phi_{9}$ series:

$$
\begin{aligned}
& { }_{10} \phi_{9}\left[\begin{array}{c}
a, q \sqrt{a},-q \sqrt{a}, b, c, a / b c, C / A q^{n}, 1 / B C q^{n}, B / A q^{n}, q^{-n} \\
\sqrt{a},-\sqrt{a}, a q / b, a q / c, b c q, 1 / C q^{n}, B C / A q^{n}, 1 / B q^{n}, 1 / A q^{n}
\end{array} ;, q\right] \\
& =\frac{(a q, b q, c q, a q / b c, A q / B, A q / C, B C q ; q)_{n}}{(A q, B q ; C q, A q / B C, a q / b, a q / c, b c q ; q)_{n}} \\
& \times_{10} \phi_{9}\left[\begin{array}{c}
A, q \sqrt{A},-q \sqrt{A}, B, C, A / B C, c / a q^{n}, 1 / b c q^{n}, b / a q^{n}, q^{-n} \\
\sqrt{A},-\sqrt{A}, A q / B, A q / C, B C q, 1 / c q^{n}, b c / a q^{n}, 1 / b q^{n}, 1 / a q^{n}
\end{array} ;, q\right] .
\end{aligned}
$$

Formula (2.10) can also be used to derive a transformation formula containing two independent bases and shifted factorials by replacing $A, B, C$ by $P^{A}$, $P^{B}, P^{C}$, setting $P=Q^{\mu}$, and letting $Q \rightarrow 1$ to get

$$
\begin{aligned}
\sum_{k=0}^{n} & \frac{\left(1-a p^{k} q^{k}\right)\left(1-b p^{k} q^{-k}\right)}{(1-a)(1-b)} \frac{(a, b ; p)_{k}(c, a / b c ; q)_{k}}{(q, a q / b ; q)_{k}(a p / c, b c p ; p)_{k}} \\
& \times \frac{(C-A-n)_{k}(-B-C-n)_{k}(\mu B-\mu A-n)_{k}(-n)_{k}}{(-\mu C-n)_{k}(\mu B+\mu C-\mu A-n)_{k}(-A-n)_{k}(-B-n)_{k}} q^{k} \\
= & \frac{(a p, b p ; p)_{n}(c q, a q / b c ; q)_{n}}{(q, a q / b ; q)_{n}(a p / c, b c p ; p)_{n}} \frac{n !(\mu A+1-\mu)_{n}(A+1-C)_{n}(B+C+1)_{n}}{(A+1)_{n}(B+1)_{n}(\mu C+1)_{n}(\mu A+1-\mu B-\mu C)_{n}} \\
& \times \sum_{k=0}^{n} \frac{(A+k+k / \mu)(B+k-k / \mu)}{A B} \frac{(A)_{k}(B)_{k}(\mu C)_{k}(\mu A-\mu B-\mu C)_{k}}{k !(\mu A+1-\mu B)_{k}(A+1-C)_{k}(B+C+1)_{k}} \\
& \times \frac{\left(c / a p^{n}, 1 / b c p^{n} ; p\right)_{k}\left(q^{-n}, b / a q^{n} ; q\right)_{k}}{\left(1 / c q^{n}, b c / a q^{n} ; q\right)_{k}\left(1 / a p^{n}, 1 / b p^{n} ; p\right)_{k}} .
\end{aligned}
$$

Similarly, replacing $a, b, c$ in (2.12) by $p^{a}, p^{b}, p^{c}$, setting $p=q^{\lambda}$ and letting $q \rightarrow 1$, we obtain the rather strange looking ${ }_{10} F_{9}$ transformation formula

$$
\begin{aligned}
& { }_{10} F_{9}\left[\begin{array}{c}
a, 1+\lambda a /(\lambda+1), 1+\lambda b /(\lambda-1), b, \lambda c, \lambda a-\lambda b-\lambda c \\
\lambda a /(\lambda+1), \lambda b /(\lambda-1), \lambda a+1-\lambda b, a+1-c, b+c+1,
\end{array}\right. \\
& \left.\begin{array}{l}
C-A-n,-B-C-n, \mu B-\mu A-n,-n \\
-\mu C-n, \mu B+\mu C-\mu A-n,-B-n,-A-n
\end{array} ; 1\right] \\
& =\frac{(a+1)_{n}(b+1)_{n}(\lambda c+1)_{n}(\lambda a+1-\lambda b-\lambda c)_{n}(\mu A+1-\mu B)_{n}(A+1-C)_{n}(B+C+1)_{n}}{(A+1)_{n}(B+1)_{n}(\mu C+1)_{n}(\mu A+1-\mu B-\mu C)_{n}(\lambda a+1-\lambda b)_{n}(a+1-c)_{n}(b+c+1)_{n}}, \\
& { }_{10} F_{9}\left[\begin{array}{c}
A, 1+\mu A /(\mu+1), 1+\mu B /(\mu-1), B, \mu C, \mu A-\mu B-\mu C, \\
\mu A /(\mu+1), \mu B /(\mu-1), \mu A+1-\mu B, A+1-C, B+C+1,
\end{array}\right. \\
& \left.\begin{array}{c}
c-a-n,-b-c-n, \lambda b-\lambda a-n,-n \\
-\lambda c-n, \lambda b+\lambda c-\lambda a-n,-b-n,-a-n
\end{array} ; 1\right] .
\end{aligned}
$$

To prove (1.11) and the transformation formulas in (1.12) first observe that, since $1-a p^{k} q^{k}=\left(1-a p^{k}\right) q^{k}+\left(1-q^{k}\right)$,

$$
\sum_{k=0}^{\infty} \frac{1-a p^{k} q^{k}}{1-a} \frac{(a ; p)_{k}(c / b ; q)_{k}}{(q ; q)_{k}(a b p ; p)_{k}} b^{k}=S_{1}+S_{2}
$$


with

$$
\begin{gathered}
S_{1}=\sum_{k=0}^{\infty} \frac{(a p ; p)_{k}(c / b ; q)_{k}}{(q ; q)_{k}(a b p ; p)_{k}}(b q)^{k} \\
S_{2}=\frac{1}{1-a} \sum_{k=1}^{\infty} \frac{(a ; p)_{k}(c / b ; q)_{k}}{(q ; q)_{k-1}(a b p ; p)_{k}} b^{k} .
\end{gathered}
$$

Employing the $q$-binomial theorem

$$
\sum_{k=0}^{\infty} \frac{(a ; q)_{k}}{(q ; q)_{k}} z^{k}=\frac{(a z ; q)_{\infty}}{(z ; q)_{\infty}}
$$

we have

$$
\begin{aligned}
S_{1} & =\frac{(a p ; p)_{\infty}}{(a b p ; p)_{\infty}} \sum_{k=0}^{\infty} \frac{(c / b ; q)_{k}}{(q ; q)_{k}}(b q)^{k} \frac{\left(a b p^{k+1} ; p\right)_{\infty}}{\left(a p^{k+1} ; p\right)_{\infty}} \\
& =\frac{(a p ; p)_{\infty}}{(a b p ; p)_{\infty}} \sum_{k=0}^{\infty} \frac{(c / b ; q)_{k}}{(q ; q)_{k}}(b q)^{k} \sum_{j=0}^{\infty} \frac{(b ; p)_{j}}{(p ; p)_{j}}\left(a p^{k+1}\right)^{j} \\
& =\frac{(a p ; p)_{\infty}}{(a b p ; p)_{\infty}} \sum_{j=0}^{\infty} \frac{(b ; p)_{j}}{(p ; p)_{j}}(a p)^{j} \sum_{k=0}^{\infty} \frac{(c / b ; q)_{k}}{(q ; q)_{k}}\left(b q p^{j}\right)^{k} \\
& =\frac{(a p ; p)_{\infty}}{(a b p ; p)_{\infty}} \sum_{j=0}^{\infty} \frac{(b ; p)_{j}\left(c q p^{j} ; q\right)_{\infty}}{(p ; p)_{j}\left(b q p^{j} ; q\right)_{\infty}}(a p)^{j}
\end{aligned}
$$

Similarly,

$$
\begin{aligned}
S_{2} & =\frac{1}{1-a} \sum_{k=0}^{\infty} \frac{(a ; p)_{k+1}(c / b ; q)_{k+1}}{(q ; q)_{k}(a b p ; p)_{k+1}} b^{k+1} \\
& =\frac{(b-c)(a p ; p)_{\infty}}{(a b p ; p)_{\infty}} \sum_{k=0}^{\infty} \frac{(c q / b ; q)_{k}\left(a b p^{k+2} ; p\right)_{\infty}}{(q ; q)_{k}\left(a p^{k+1} ; p\right)_{\infty}} b^{k} \\
& =\frac{(b-c)(a p ; p)_{\infty}}{(a b p ; p)_{\infty}} \sum_{k=0}^{\infty} \frac{(c q / b ; q)_{k}}{(q ; q)_{k}} b^{k} \sum_{j=0}^{\infty} \frac{(b p ; p)_{j}}{(p ; p)_{j}}\left(a p^{k+1}\right)^{j} \\
& =\frac{(b-c)(a p ; p)_{\infty}}{(a b p ; p)_{\infty}} \sum_{j=0}^{\infty} \frac{(b p ; p)_{j}}{(p ; p)_{j}}(a p)^{j} \sum_{k=0}^{\infty} \frac{(c q / b ; q)_{k}}{(q ; q)_{k}}\left(b p^{j}\right)^{k} \\
& =\frac{(b-c)(a p ; p)_{\infty}}{(1-b)(a b p ; p)_{\infty}} \sum_{j=0}^{\infty} \frac{(b ; p)_{j}\left(c q p^{j} ; q\right)_{\infty}}{(p ; p)_{j}\left(b q p^{j} ; q\right)_{\infty}}(a p)^{j}
\end{aligned}
$$

Hence

$$
\begin{gathered}
S_{2}=\frac{b-c}{1-b} S_{1}, \\
S_{1}+S_{2}=\frac{1-c}{1-b} S_{1},
\end{gathered}
$$


which, in combination with (2.18) and (2.19), gives (1.12). Formula (1.11) follows by setting $c=1$ in (1.12).

\section{INVERSE MATRICES AND EXTENSIONS OF EULER'S TRANSFORMATION FORMULA}

By switching $p$ and $q$ in (2.3) and replacing $n, a, b$ and $k$ by $n-m$, $a p^{m} q^{m}, b p^{-m} q^{m}$ and $j-m$, we find that

$$
\sum_{j=m}^{n} a_{n j} b_{j m}=\delta_{n, m}
$$

with

$$
\begin{gathered}
a_{n j}=\frac{(-1)^{n+j}\left(1-a p^{j} q^{j}\right)\left(1-b p^{-j} q^{j}\right)\left(a q p^{n}, b q p^{-n} ; q\right)_{n-1}}{(p ; p)_{n-j}\left(a q p^{n}, b q p^{-n} ; q\right)_{j}\left(b p^{1-2 n} / a ; p\right)_{n-j}}, \\
b_{j m}=\frac{\left(a p^{m} q^{m}, b p^{-m} q^{m} ; q\right)_{j-m}}{\left(p, a p^{1+2 m} / b ; p\right)_{j-m}}\left(-\frac{a}{b} p^{1+2 m}\right)^{j-m} p^{2\left(\begin{array}{c}
j-m \\
2
\end{array}\right)},
\end{gathered}
$$

which shows that the triangular matrix $A=\left(a_{n j}\right)$ is inverse to the triangular matrix $B=\left(b_{j m}\right)$. Also see Bressoud [8, $\left.\S 4\right]$. The Al-Salam and Verma inverse matrices $H$ and $G$ are the $b \rightarrow 0$ limit cases of $A$ and $B$.

Computing the $j k$ th term of the product matrix $B A$, we get

$$
\begin{aligned}
& \sum_{n=0}^{j-k} \frac{\left(1-a p^{k} q^{k}\right)\left(1-b p^{-k} q^{k}\right)\left(a p^{k+n} q^{k+1}, b p^{-k-n} q^{k+1} ; q\right)_{j-k-1}}{(p ; p)_{n}(p ; p)_{j-k-n}\left(a p^{2 k+n} / b ; p\right)_{j-k+1}} \\
& \quad \times\left(1-\frac{a}{b} p^{2 k+2 n}\right)(-1)^{n} p^{n(j-k-1)+\left(\begin{array}{c}
j-k-n \\
2
\end{array}\right)}=\delta_{j, k},
\end{aligned}
$$

which, on replacing $j, n, a, b$, respectively, by $n+k, k, a p^{-k} q^{-k-1}$, $b p^{k} q^{-k-1}$, gives the following extension of (1.5)

$$
\left(1-\frac{a}{q}\right)\left(1-\frac{b}{q}\right) \sum_{k=0}^{n} \frac{\left(a p^{k}, b p^{-k} ; q\right)_{n-1}\left(1-a p^{2 k} / b\right)}{(p ; p)_{k}(p ; p)_{n-k}\left(a p^{k} / b ; p\right)_{n+1}}(-1)^{k} p^{k(n-1)+\left(\frac{n-k}{2}\right)}=\delta_{n, 0} \text {. }
$$

We can now use this bibasic sum to derive an extension of the Al-Salam and Verma bibasic extension (1.7) of Euler's transformation formula (1.6). Replace $n$ in (3.5) by $j$, multiply both sides by $B_{n+j}(-x)^{n+j}(a / b)^{j} p^{j(j+1) / 2}$, sum from $j=0$ to $\infty$, change the order of summation and then replace $k$ by $k-n$ and $j$ by $j+k-n$ to get

$$
\begin{aligned}
B_{n}(-x)^{n}= & \left(1-\frac{a}{q}\right)\left(1-\frac{b}{q}\right) \sum_{k=n}^{\infty} \frac{1-a p^{2 k-2 n} / b}{(p ; p)_{k-n}}(-x)^{k} \\
& \times \sum_{j=0}^{\infty} \frac{\left(a p^{k-n}, b p^{n-k} ; q\right)_{j+k-n-1}}{(p ; p)_{j}\left(a p^{k-n} / b ; p\right)_{j+k-n+1}}\left(-\frac{a}{b}\right)^{j+k-n} \\
& \times x^{j} B_{j+k} p^{(k-n)(j+k-n-1)+\left(\begin{array}{l}
j \\
2
\end{array}\right)+\left(\begin{array}{c}
j+k-n+1 \\
2
\end{array}\right)}
\end{aligned}
$$


Now replace $a$ by $a p^{n} q^{n+1}, b$ by $b p^{-n} q^{n+1}$, multiply both sides by $A_{n} w^{n}$, then sum from $n=0$ to $\infty$ to obtain

$$
\begin{aligned}
\sum_{n=0}^{\infty} A_{n} B_{n}(-x w)^{n}= & \sum_{k=0}^{\infty} \frac{\left(a q p^{k}, b q p^{-k} ; q\right)_{k-1}}{\left(a p^{k} / b ; p\right)_{k}}(-x)^{k} \\
& \times \sum_{n=0}^{k} \frac{\left(1-a p^{n} q^{n}\right)\left(1-b p^{-n} q^{n}\right)\left(a p^{k} / b ; p\right)_{n}}{(p ; p)_{k-n}\left(a q p^{k}, b q p^{-k} ; q\right)_{n}} A_{n} w^{n} \\
& \times \sum_{j=0}^{\infty} \frac{\left(a p^{k} q^{k}, b p^{-k} q^{k} ; q\right)_{j}}{(p ; p)_{j}\left(a p^{2 k+1} / b ; p\right)_{j}}\left(-\frac{a}{b} p^{2 n}\right)^{j+k-n} \\
& \times B_{j+k} x^{j} p^{(k-n)(j+k-n-1)+\left(\begin{array}{l}
j \\
2
\end{array}\right)+\left(\begin{array}{c}
j+k-n+1 \\
2
\end{array}\right) .}
\end{aligned}
$$

We have written this formula in a form so that it tends directly to (1.7) as $b \rightarrow 0$. By replacing $A_{n}, B_{n}, x, w$ by suitable multiples, formula (3.7) can be written in an equivalent form which tends to (1.7) as $b \rightarrow \infty$. Also, by replacing $A_{n}, B_{n}, x, w$ by $A_{n} p^{2\left(\begin{array}{l}n \\ 2\end{array}\right)}, B_{n} p^{-2\left(\begin{array}{l}n \\ 2\end{array}\right)},-b x / a, a w / b$, respectively, (3.7) can be written in the simpler looking equivalent form

$$
\begin{aligned}
\sum_{n=0}^{\infty} A_{n} B_{n} x^{n} w^{n}= & \sum_{k=0}^{\infty} \frac{\left(a q p^{k}, b q p^{-k} ; q\right)_{k-1}}{\left(p, a p^{k} / b ; p\right)_{k}}(-x)^{k} p^{k+\left(\begin{array}{l}
k \\
2
\end{array}\right)} \\
& \times \sum_{n=0}^{k} \frac{\left(1-a q^{n} p^{n}\right)\left(1-b q^{n} p^{-n}\right)\left(p^{-k}, a p^{k} / b ; p\right)_{n}}{\left(a q p^{k}, b q p^{-k} ; q\right)_{n}} A_{n} w^{n} \\
& \times \sum_{j=0}^{\infty} \frac{\left(a q^{k} p^{k}, b q^{k} p^{-k} ; q\right)_{j}}{\left(p, a p^{2 k+1} / b ; p\right)_{j}} B_{j+k} x^{j} p^{j}
\end{aligned}
$$

As in our derivation of (2.4) from (1.14), we find that both (3.7) and (3.8) yield the expansion formula

$$
\begin{aligned}
\sum_{n=0}^{\infty} A_{n} B_{n} x^{n} w^{n}= & \sum_{k=0}^{\infty} \frac{(\lambda a+1+\lambda k)_{k-1}(\lambda b+1-\lambda k)_{k-1}}{k !(a+k-b)_{k}}(-x)^{k} \\
& \times \sum_{n=0}^{k} \frac{(\lambda a+n+\lambda n)(\lambda b+n-\lambda n)(-k)_{n}(a+k-b)_{n}}{(\lambda a+1+\lambda k)_{n}(\lambda b+1-\lambda k)_{n}} A_{n} w^{n} \\
& \times \sum_{j=0}^{\infty} \frac{(\lambda a+k+\lambda k)_{j}(\lambda b+k-\lambda k)_{j}}{j !(a+2 k+1-b)_{j}} B_{j+k} x^{j}
\end{aligned}
$$

Here, as elsewhere, either the parameters and variables are assumed to be such that the (multiple) series converge absolutely, or the series are considered to be formal power series in the variables $x$ and $w$.

In [23] Srivastava repeated the Al-Salam and Verma proof of (1.5) in a form corresponding to inverting the base $p$ and used this formula to derive a multivariable generalization of the Al-Salam and Verma expansion (1.7) which generalized several of his earlier results. Unfortunately, he did not observe that his 
formula [23, (10)] was, in fact, a special case of (1.7), as can be easily seen by using the observation that, for arbitrary positive integers $m_{1}, \ldots, m_{r}$,

$$
\begin{aligned}
& \sum_{k_{1}, \ldots, k_{r}=0}^{\infty} \Lambda\left(k_{1}, \ldots, k_{r}\right) z_{1}^{k_{1}} \cdots z_{r}^{k_{r}} \\
&=\sum_{M=0}^{\infty} \sum_{\substack{k_{1} m_{1}+\cdots+k_{r} m_{r}=M \\
k_{1}, \ldots, k_{r} \geq 0}} \Lambda\left(k_{1}, \ldots, k_{r}\right) z_{1}^{k_{1}} \cdots z_{r}^{k_{r} .}
\end{aligned}
$$

Applying this observation to (3.8) with

$$
A_{n}=\sum_{\substack{k_{1} m_{1}+\cdots+k_{r}, m_{r}=n \\ k_{1}, \ldots, k_{r} \geq 0}} \Lambda\left(k_{1}, \ldots, k_{r}\right) z_{1}^{k_{1}} \cdots z_{r}^{k_{r}}, \quad B_{n}=\Omega_{n},
$$

and letting

$$
M=M\left(m_{1}, \ldots, m_{r}, k_{1}, \ldots, k_{r}\right)=k_{1} m_{1}+\cdots+k_{r} m_{r},
$$

where $m_{1}, \ldots, m_{r}$ are (fixed) positive integers, we obtain the multivariable expansion formula

$$
\begin{aligned}
& \sum_{k_{1}, \ldots, k_{r}=0}^{\infty} \Lambda\left(k_{1}, \ldots, k_{r}\right) \Omega_{k_{1} m_{1}+\cdots+k_{r} m_{r}}\left(x^{m_{1}} w^{m_{1}} z_{1}\right)^{k_{1}} \cdots\left(x^{m_{r}} w^{m_{r}} z_{r}\right)^{k_{r}} \\
& =\sum_{n=0}^{\infty} \frac{\left(a q p^{n}, b q p^{-n} ; q\right)_{n-1}}{\left(p, a p^{n} / b ; p\right)_{n}}(-x)^{n} p^{n+\left(\begin{array}{l}
n \\
2
\end{array}\right)} \\
& \quad \times \sum_{j=0}^{\infty} \frac{\left(a q^{n} p^{n}, b q^{n} p^{-n} ; q\right)_{j}}{\left(p, a p^{2 n+1} / b ; p\right)_{j}} \Omega_{j+n} x^{j} p^{j} \\
& \quad \times \sum_{\substack{k_{1} m_{1}+\cdots+k_{r} m_{r} \leq n \\
k_{1} \ldots, k_{r} \geq 0}} \frac{\left(1-a q^{M} p^{M}\right)\left(1-b q^{M} p^{-M}\right)\left(p^{-n}, a p^{n} / b ; p\right)_{M}}{\left(a q p^{n}, b q p^{-n} ; q\right)_{M}} \\
& \quad \times \Lambda\left(k_{1}, \ldots, k_{r}\right)\left(w^{m_{1}} z_{1}\right)^{k_{1}} \cdots\left(w^{m_{r}} z_{r}\right)^{k_{r}},
\end{aligned}
$$

which extends [23, (10)] and is equivalent to (3.8). Use of (3.11) in (3.9) gives a limit case of (3.13).

Analogous to the $q$-analogue of Lagrange inversion for $x /(1-x)^{b+1}$ in Gessel and Stanton [15, Theorem 3.7], the fact that $B=A^{-1}$ is equivalent to the following bibasic Lagrange inversion result:

If

$$
G_{n}(x)=\sum_{j=n}^{\infty} b_{j n} x^{j}
$$

where $b_{j n}$ is as defined in (3.3), and if

$$
f(x)=\sum_{j=0}^{\infty} f_{j} x^{j}=\sum_{n=0}^{\infty} c_{n} G_{n}(x),
$$


then

$$
f_{j}=\sum_{n=0}^{j} b_{j n} c_{n}
$$

and, vice versa,

$$
c_{n}=\sum_{j=0}^{n} a_{n j} f_{j},
$$

where $a_{n j}$ is as defined in (3.2).

For some applications of special cases of the above result to identities of Rogers-Ramanujan type and to quadratic and cubic transformations, see [15, 16].

\section{EXTENSIONS OF THE FiELdS AND WIMP EXPANSION}

Verma [25] showed that the Fields and Wimp expansion (1.15) is a special case of the expansion

$$
\begin{aligned}
\sum_{n=0}^{\infty} A_{n} B_{n} \frac{(x w)^{n}}{n !}= & \sum_{n=0}^{\infty} \frac{(-x)^{n}}{n !(\gamma+n)_{n}} \sum_{k=0}^{\infty} \frac{(\alpha)_{n+k}(\beta)_{n+k}}{k !(\gamma+2 n+1)_{k}} B_{n+k} x^{k} \\
& \times \sum_{j=0}^{n} \frac{(-n)_{j}(n+\gamma)_{j}}{j !(\alpha)_{j}(\beta)_{j}} A_{j} w^{j}
\end{aligned}
$$

and derived the $q$-analogue

$$
\begin{aligned}
& \sum_{n=0}^{\infty} A_{n} B_{n} \frac{(x w)^{n}}{(q ; q)_{n}} \\
& \quad=\sum_{n=0}^{\infty} \frac{(-x)^{n}}{\left(q, \gamma q^{n} ; q\right)_{n}} q^{\left(\begin{array}{l}
n \\
2
\end{array}\right)} \sum_{k=0}^{\infty} \frac{(\alpha, \beta ; q)_{n+k}}{\left(q, \gamma q^{2 n+1} ; q\right)_{k}} B_{n+k} x^{k} \\
& \quad \cdot \sum_{j=0}^{n} \frac{\left(q^{-n}, \gamma q^{n} ; q\right)_{j}}{(q, \alpha, \beta ; q)_{j}} A_{j}(w q)^{j}
\end{aligned}
$$

where we have corrected a slight misprint.

To derive bibasic extensions of (4.2), first observe that

$$
\begin{aligned}
& \sum_{j=0}^{m} \frac{\left(1-\gamma p^{r+j} q^{r+j}\right)\left(1-\sigma p^{r+j} q^{-r-j}\right)}{\left(1-\gamma p^{r} q^{r}\right)\left(1-\sigma p^{r} q^{-r}\right)} \frac{\left(\gamma p^{r} q^{r}, \sigma p^{r} q^{-r} ; p\right)_{j}}{\left(q, \gamma \sigma^{-1} q^{2 r+1} ; q\right)_{j}} \\
& \quad \times \frac{\left(q^{-m}, \gamma \sigma^{-1} q^{2 r+m} ; q\right)_{j}}{\left(\gamma p^{r+1} q^{r+m}, \sigma p^{r+1} q^{-r-m} ; p\right)_{j}} q^{j}=\delta_{m, 0}
\end{aligned}
$$


by (1.14), and hence, if $C_{r, m}$ are complex numbers such that $C_{r, 0}=1$ for $r=0,1,2, \ldots$,

$$
\begin{aligned}
B_{r} x^{r} & =\sum_{m=0}^{\infty} \frac{1-\gamma \sigma^{-1} q^{2 r+2 m}}{1-\gamma \sigma^{-1} q^{2 r}} \frac{\left(\gamma \sigma^{-1} q^{2 r} ; q\right)_{m}\left(\gamma p q^{r}, \sigma p q^{-r} ; p\right)_{r}}{(q ; q)_{m}\left(\gamma p q^{r+m}, \sigma p q^{-r-m} ; p\right)_{r}} \\
\times q^{-m r} B_{r+m} C_{r, m} x^{r+m} \delta_{m, 0} & \\
= & \sum_{k=0}^{\infty} \sum_{n=r}^{\infty} \frac{\left(1-\gamma p^{n} q^{n}\right)\left(1-\sigma p^{n} q^{-n}\right)\left(1-\gamma \sigma^{-1} q^{2 n+2 k}\right)\left(\gamma \sigma^{-1} q^{n+r+1} ; q\right)_{n+k-r-1}}{(q ; q)_{k}(q ; q)_{n}\left(\gamma p q^{n+k}, \sigma p q^{-n-k} ; p\right)_{n}} \\
& \times\left(\gamma p q^{r}, \sigma p q^{-r} ; p\right)_{n-1}\left(q^{-n} ; q\right)_{r}(-1)^{n} B_{n+k} C_{r, n+k-r} x^{n+k} q^{n(1+r-n-k)+\left(\begin{array}{l}
n \\
2
\end{array}\right)}
\end{aligned}
$$

by letting $j=n-r$ and $m=n+k-r$. Now multiply both sides by $A_{r} w^{r} /(q ; q)_{r}$ and sum over $r$ from $r=0$ to $\infty$ to obtain the bibasic expansion formula

$$
\begin{aligned}
\sum_{n=0}^{\infty} A_{n} B_{n} \frac{(x w)^{n}}{(q ; q)_{n}}= & \sum_{n=0}^{\infty} \frac{\left(1-\gamma p^{n} q^{n}\right)\left(1-\sigma p^{n} q^{-n}\right)}{(q ; q)_{n}}(-x)^{n} q^{n+\left(\begin{array}{l}
n \\
2
\end{array}\right)} \\
& \times \sum_{k=0}^{\infty} \frac{1-\gamma \sigma^{-1} q^{2 n+2 k}}{(q ; q)_{k}\left(\gamma p q^{n+k}, \sigma p q^{-n-k} ; p\right)_{n}} B_{n+k} x^{k} \\
& \times \sum_{j=0}^{n} \frac{\left(q^{-n} ; q\right)_{j}\left(\gamma \sigma^{-1} q^{n+j+1} ; q\right)_{n+k-j-1}}{(q ; q)_{j}} \\
& \times\left(\gamma p q^{j}, \sigma p q^{-j} ; p\right)_{n-1} A_{j} C_{j, n+k-j} w^{j} q^{n(j-n-k)},
\end{aligned}
$$

where $C_{j, 0}=1$ for $j=0,1, \ldots$. Similarly, formula (3.7) can be extended by replacing $B_{j+k}$ in (3.6) by $B_{j+k} C_{n, j+k-n}$ with $C_{n, 0}=1$ for $n=1,2, \ldots$.

When $p=q$ and $C_{j, m} \equiv 1,(4.5)$ reduces to an expansion equivalent to

$$
\begin{aligned}
\sum_{n=0}^{\infty} & A_{n} B_{n} \frac{(x w)^{n}}{(q ; q)_{n}}=\sum_{n=0}^{\infty} \frac{\left(\sigma, \gamma q^{n+1} / \sigma, \alpha, \beta ; q\right)_{n}}{\left(q, \gamma q^{n} ; q\right)_{n}}\left(\frac{x}{\sigma}\right)^{n} \\
& \times \sum_{k=0}^{\infty} \frac{\left(\gamma q^{2 n} / \sigma, q^{n+1} \sqrt{\gamma / \sigma},-q^{n+1} \sqrt{\gamma / \sigma}, 1 / \sigma, \alpha q^{n}, \beta q^{n} ; q\right)_{k}}{\left(q, q^{n} \sqrt{\gamma / \sigma},-q^{n} \sqrt{\gamma / \sigma}, \gamma q^{2 n+1} ; q\right)_{k}} B_{n+k} x^{k} \\
& \times \sum_{j=0}^{n} \frac{\left(q^{-n}, \gamma q^{n} ; q\right)_{j}}{\left(q, \gamma q^{n+1} / \sigma, q^{1-n} / \sigma, \alpha, \beta ; q\right)_{j}} A_{j}(w q)^{j} .
\end{aligned}
$$


Verma's expansion (4.2) is the $\sigma \rightarrow \infty$ limit case of (4.6). For basic hypergeometric series, (4.6) gives the expansion

$$
\begin{aligned}
r_{r+t} \phi_{s+u}\left[\begin{array}{l}
\left.a_{R}, c_{T} ; q, x w\right] \\
b_{S}, d_{U}
\end{array}\right. \\
=\sum_{n=0}^{\infty} \frac{\left(c_{T}, e_{K}, \sigma, \gamma q^{n+1} / \sigma ; q\right)_{n}}{\left(q, d_{U}, f_{M}, \gamma q^{n} ; q\right)_{n}}\left(\frac{x}{\sigma}\right)^{n}\left[(-1)^{n} q^{\left(\begin{array}{l}
n \\
2
\end{array}\right)}\right]^{m+u-k-t} \\
\quad \times_{k+t+4} \phi_{m+u+3}\left[\begin{array}{c}
\gamma q^{2 n} / \sigma, q^{n+1} \sqrt{\gamma / \sigma},-q^{n+1} \sqrt{\gamma / \sigma}, \sigma^{-1}, c_{T} q^{n}, e_{K} q^{n} \\
q^{n} \sqrt{\gamma / \sigma},-q^{n} \sqrt{\gamma / \sigma}, \gamma q^{2 n+1}, d_{U} q^{n}, f_{M} q^{n}
\end{array}\right. \\
\left.\quad q, x q^{n(m+u-k-t)}\right] \\
\quad \times_{m+r+2} \phi_{k+s+2}\left[\begin{array}{c}
q^{-n}, \gamma q^{n}, a_{R}, f_{M} \\
\gamma q^{n+1} / \sigma, q^{1-n} / \sigma, b_{S}, e_{K}
\end{array} ; q, w q\right],
\end{aligned}
$$

where we used a contracted notation analogous to that used in (1.15). This formula is a $q$-analogue of a generalization of the Fields and Wimp expansion (1.15). Some other expansions are given in Fields and Ismail [10]. The multivariable expansion formula which follows by using (3.11) in (4.5) and its limit cases (see, e.g., [18 and 22]) will be omitted.

In a subsequent paper, we shall use a special case of (4.7) to derive a $q$ analogue of the inequality used by de Branges [7] in his proof of the Bieberbach conjecture. Also see [5 and 12].

\section{QUADRATIC, CUBIC, AND QUARTIC SUMMATION FORMULAS}

One of the advantages of (1.14) is that by setting $p=q^{2}, q^{3}, \ldots$ or $q=$ $p^{2}, p^{3}, \ldots$ we obtain quadratic, cubic, ... summation formulas which can then be used to derive other quadratic, cubic,... summation formulas.

In a letter to Gessel and Stanton, Gosper stated the following nonterminating version of the quadratic summation formula (1.4) in Gessel and Stanton [15]

$$
\begin{array}{r}
\sum_{n=0}^{\infty}\left(1-a b c q^{3 n+1}\right) \frac{(a b c q, d, q / d ; q)_{n}\left(b c q, a c q, a b q ; q^{2}\right)_{n}}{\left(q^{2}, a b c q^{3} / d, a b c d q^{2} ; q^{2}\right)_{n}(a q, b q, c q ; q)_{n}} q^{n} \\
=\frac{(a b c q ; q)_{\infty}\left(a d q, a q^{2} / d ; q^{2}\right)_{\infty}}{(a q ; q)_{\infty}\left(a b c q^{3} / d, a b c d q^{2} ; q^{2}\right)_{\infty}} \\
\quad \times\left(\frac{(1 / c ; q)_{\infty}\left(b d q, b q^{2} / d ; q^{2}\right)_{\infty}}{(b q ; q)_{\infty}\left(q / c d, d / c ; q^{2}\right)_{\infty}}+\frac{(d, q / d ; q)_{\infty}\left(b c q ; q^{2}\right)_{\infty}}{(b q, c q ; q)_{\infty}\left(q^{2} ; q^{2}\right)_{\infty}}\right. \\
\left.\quad \times \sum_{n=0}^{\infty} \frac{\left(q / b c ; q^{2}\right)_{n}}{\left(d / c, q / c d ; q^{2}\right)_{n+1}}(-b)^{n}\left(\frac{q^{n}}{c}\right)^{n+1}\right)
\end{array}
$$


DIBASIC SERIES

271

(continued)

$$
\begin{aligned}
& -a \frac{(a b c q, d, q / d ; q)_{\infty}\left(b c q, a c q, a b q ; q^{2}\right)_{\infty}}{\left(q^{2}, a b c q^{3} / d, a b c d q^{2} ; q^{2}\right)_{\infty}(a q, b q, c q ; q)_{\infty}} \\
& -\sum_{n=0}^{\infty} \frac{\left(a d q, a q^{2} / d ; q^{2}\right)_{n}}{\left(a b q, a c q ; q^{2}\right)_{n+1}} q^{2 n+1} .
\end{aligned}
$$

The author observed that the right side of (5.1) could be simplified to give the formula

$$
\begin{aligned}
\sum_{n=0}^{\infty} & \left(1-a b c q^{3 n+1}\right) \frac{(a b c q, d, q / d ; q)_{n}\left(b c q, a c q, a b q ; q^{2}\right)_{n}}{\left(q^{2}, a b c q^{3} / d, a b c d q^{2} ; q^{2}\right)_{n}(a q, b q, c q ; q)_{n}} q^{n} \\
= & \frac{(a b c q, 1 / c ; q)_{\infty}\left(a d q, a q^{2} / d, b d q, b q^{2} / d ; q^{2}\right)_{\infty}}{(a q, b q ; q)_{\infty}\left(a b c q^{3} / d, a b c d q^{2}, q / c d, d / c ; q^{2}\right)_{\infty}} \\
& -a q \frac{(a b c q, d, q / d ; q)_{\infty}\left(a b q^{3}, a c q^{3}, b c q, a q^{2} / c, 1 / a c q ; q^{2}\right)_{\infty}}{(a q, b q, c q ; q)_{\infty}\left(q^{2}, a b c q^{3} / d, a b c d q^{2}, q / c d, d / c ; q^{2}\right)_{\infty}} \\
& \times{ }_{3} \phi_{2}\left[\begin{array}{c}
a d q, a q^{2} / d, a b q \\
a q^{2} / c, a b q^{3}
\end{array} q^{2}, \frac{q}{a c}\right]
\end{aligned}
$$

by using the transformation formula

$$
\begin{array}{r}
{ }_{3} \phi_{2}\left[\begin{array}{c}
a d q, a q^{2} / d, a b q \\
a q^{2} / c, a b q^{3}
\end{array} q^{2}, \frac{q}{a c}\right]=\frac{\left(q / c d, d / c ; q^{2}\right)_{\infty}}{\left(a q^{2} / c, 1 / a c q ; q^{2}\right)_{\infty}} \\
\times\left({ }_{3} \phi_{2}\left[\begin{array}{c}
a d q, a q^{2} / d, q^{2} \\
a c q^{3}, a b q^{3}
\end{array} ; q^{2}, q^{2}\right]\right. \\
-\frac{\left(a d q, a q^{2} / d ; q^{2}\right)_{\infty}}{a c q(1-d / c)(1-q / c d)\left(a b q^{3}, a c q^{3} ; q^{2}\right)_{\infty}} \\
\left.\times{ }_{2} \phi_{2}\left[\begin{array}{c}
q^{2}, q / b c \\
d q^{2} / c, q^{3} / c d
\end{array} ; q^{2}, \frac{b q^{2}}{c}\right]\right),
\end{array}
$$

which follows from formula (10.2) in Sears [20] by applying Heine's

$$
{ }_{2} \phi_{1}\left[\begin{array}{c}
a, b \\
c
\end{array} ; q, x\right]=\frac{(a x, b ; q)_{\infty}}{(c, x ; q)_{\infty}}{ }_{2} \phi_{1}\left[\begin{array}{c}
c / b, x \\
a x
\end{array} ; q, b\right]
$$

and Jackson's [17]

$$
{ }_{2} \phi_{1}\left[\begin{array}{c}
a, b \\
c
\end{array} ; q, x\right]=\frac{(a x ; q)_{\infty}}{(x ; q)_{\infty}}{ }_{2} \phi_{2}\left[\begin{array}{c}
a, c / b \\
a x, c
\end{array} ; q, b x\right]
$$

transformation formulas.

We shall show how the special case

(5.6) $\sum_{k=0}^{n} \frac{\left(1-a q^{3 k}\right)\left(1-b q^{-k}\right)}{(1-a)(1-b)} \frac{(a, b ; q)_{k}\left(q^{-2 n}, a q^{2 n} / b ; q^{2}\right)_{k}}{\left(q^{2}, a q^{2} / b ; q^{2}\right)_{k}\left(a q^{2 n+1}, b q^{1-2 n} ; q\right)_{k}} q^{2 k}=\delta_{n, 0}$ 
of (1.14) can be used to prove (5.2). For any sequence $A_{n},(5.6)$ gives (5.7)

$$
\begin{aligned}
A_{0}= & \sum_{n=0}^{\infty} \frac{\left(a / b ; q^{2}\right)_{n}(1 / b ; q)_{2 n}}{\left(q^{2} ; q^{2}\right)_{n}(a q ; q)_{2 n}} A_{n} \\
& \times \sum_{k=0}^{n} \frac{\left(1-a q^{3 k}\right)\left(1-b q^{-k}\right)}{(1-a)(1-b)} \frac{(a, b ; q)_{k}\left(q^{-2 n}, a q^{2 n} / b ; q^{2}\right)_{k}}{\left(q^{2}, a q^{2} / b ; q^{2}\right)_{k}\left(a q^{2 n+1}, b q^{1-2 n} ; q\right)_{k}} q^{2 k} \\
= & \sum_{k=0}^{\infty} \frac{\left(1-a q^{3 k}\right)\left(1-b q^{-k}\right)}{(1-a)(1-b)} \frac{(a, b, 1 / b ; q)_{k}\left(a / b ; q^{2}\right)_{2 k}}{\left(q^{2}, a q^{2} / b ; q^{2}\right)_{k}(a q ; q)_{3 k}} b^{-k} q^{k+\left(\begin{array}{l}
k \\
2
\end{array}\right)} \\
& \times \sum_{n=0}^{\infty} \frac{\left(a q^{4 k} / b, q^{k} / b, q^{k+1} / b ; q^{2}\right)_{n}}{\left(q^{2}, a q^{3 k+2}, a q^{3 k+1} ; q^{2}\right)_{n}} A_{n+k}
\end{aligned}
$$

provided that the series converge absolutely. By taking

$$
A_{n}=\frac{\left(q^{2} \sqrt{a / b},-q^{2} \sqrt{a / b}, c, d, a^{2} q / c d ; q^{2}\right)_{n}}{\left(\sqrt{a / b},-\sqrt{a / b}, a q^{2} / b c, a q^{2} / b d, c d q / a b ; q^{2}\right)_{n}} q^{2 n}
$$

(5.7) gives

$$
\begin{aligned}
1= & \sum_{k=0}^{\infty} \frac{\left(1-a q^{3 k}\right)\left(1-b q^{-k}\right)}{(1-a)(1-b)} \frac{(a, b, 1 / b ; q)_{k}\left(a / b ; q^{2}\right)_{2 k}}{\left(q^{2}, a q^{2} / b ; q^{2}\right)_{k}(a q ; q)_{3 k}} \\
& \times \frac{\left(q^{2} \sqrt{a / b},-q^{2} \sqrt{a / b}, c, d, a^{2} q / c d ; q^{2}\right)_{k}}{\left(\sqrt{a / b},-\sqrt{a / b}, a q^{2} / b c, a q^{2} / b d, c d q / a b ; q^{2}\right)_{k}} b^{-k} q^{3 k+\left(\begin{array}{l}
k \\
2
\end{array}\right)} \\
& \times{ }_{8} W_{7}\left(a q^{4 k} / b ; a^{2} q^{2 k+1} / c d, c q^{2 k}, d q^{2 k}, q^{k} / b, q^{k+1} / b ; q^{2}, q^{2}\right)
\end{aligned}
$$

where we let

$$
\begin{aligned}
& { }_{8} W_{7}(a ; b, c, d, e, f ; q, z) \\
& \quad={ }_{8} \phi_{7}\left[\begin{array}{c}
a, q \sqrt{a},-q \sqrt{a}, b, c, d, e, f \\
\sqrt{a},-\sqrt{a}, a q / b, a q / c, a q / d, a q / e, a q / f
\end{array} ; q, z\right] .
\end{aligned}
$$

Applying Bailey's nonterminating extension of Jackson's summation formula [21, (IV.15), p. 248]

$$
\begin{aligned}
&{ }_{8} W_{7}(a ; b, c, d, e, f ; q, q) \\
&= \frac{(a q, b / a, a q / d e, a q / c e, a q / c d, a q / c f, a q / d f, a q / e f ; q)_{\infty}}{(a q / c, a q / d, a q / e, a q / f, b c / a, b d / a, b e / a, b f / a ; q)_{\infty}} \\
&-\frac{(b q / c, b q / d, b q / e, b q / f, a q, c, d, e, f, b / a ; q)_{\infty}}{\left(b^{2} q / a, b c / a, b d / a, b e / a, b f / a, a q / c, a q / d, a q / e, a q / f, a / b ; q\right)_{\infty}} \\
& \times{ }_{8} W_{7}\left(b^{2} / a ; b, b c / a, b d / a, b e / a, b f / a ; q, q\right),
\end{aligned}
$$


where $a^{2} q=b c d e f$, we find from (5.9) that

$$
\begin{aligned}
1= & \frac{(a q / c, a q / d ; q)_{\infty}\left(a b q, a b q / c d, a q^{2} / b, a q^{2} / b c d ; q^{2}\right)_{\infty}}{(a q, a q / c d ; q)_{\infty}\left(a b q / c, a b q / d, a q^{2} / b c, a q^{2} / b d ; q^{2}\right)_{\infty}} \\
& \times \sum_{k=0}^{\infty} \frac{\left(1-a q^{3 k}\right.}{1-a} \frac{(a, b, q / b ; q)_{k}\left(c, d, a^{2} q / c d ; q^{2}\right)_{k}}{\left(q^{2}, a q^{2} / d, a b q ; q^{2}\right)_{k}(a q / c, a q / d, c d / a ; q)_{k}} q^{k} \\
& -\frac{\left(a^{2} b q^{2} / c d, 1 / b ; q\right)_{\infty}\left(c, d, a b q / c d, a q^{2} / b, a^{2} q^{3} / c^{2} d, a^{2} q^{3} / c d^{2} ; q^{2}\right)_{\infty}}{(a q / c d, a q ; q)_{\infty}\left(a b q / c, a b q / d, c d / a b q, a q^{2} / b c, a q^{2} / b d, \mu q^{2} ; q^{2}\right)_{\infty}} \\
& \times \sum_{j=0}^{\infty} \frac{\left(\mu, q \sqrt{\mu},-q \sqrt{u}, a b q / d, a b q / c, a^{2} q / c d, a q / c d, a q^{2} / c d ; q^{2}\right)_{j}}{\left(q^{2}, \sqrt{\mu},-\sqrt{\mu}, a^{2} q^{3} / c^{2} d, a^{2} q^{3} / c d^{2}, a b q^{3} / c d, a^{2} b q^{2} / c d, a^{2} b q^{3} / c d ; q^{2}\right)_{j}} q^{2 j} \\
& \times \sum_{k=0}^{\infty} \frac{\left(1-a q^{3 k}\right)\left(1-b q^{-k}\right)}{(1-a)(1-b)} \frac{(a, b ; q)_{k}\left(a^{2} q^{1+2 j} / c d, c d q^{-2 j} / a b q ; q^{2}\right)_{k}}{\left(q^{2}, a q^{2} / b ; q^{2}\right)_{k}\left(c d q^{-2 j} / a, a^{2} b q^{2+2 j} / c d ; q\right)_{k}} q^{2 k}
\end{aligned}
$$

with $\mu=a^{3} b q^{2} / c^{2} d^{2}$. The case $n=\infty$ of (1.14) shows that the sum over $k$ in (5.12) equals

$$
\frac{(a q, b q ; q)_{\infty}\left(a^{2} q^{2 j+3} / c d, c d q^{1-2 j} / a b ; q^{2}\right)_{\infty}}{\left(q^{2}, a q^{2} / b ; q^{2}\right)_{\infty}\left(c d q^{-2 j} / a, a^{2} b q^{2 j+2} / c d ; q\right)_{\infty}}
$$

and so the double sum over $j$ and $k$ in (5.12) equals

$$
\begin{aligned}
& \frac{(a q, b q ; q)_{\infty}\left(a^{2} q^{3} / c d, c d q / a b ; q^{2}\right)_{\infty}}{\left(a^{2}, a q^{2} / b ; q^{2}\right)_{\infty}\left(c d / a, a^{2} b q^{2} / c d ; q\right)_{\infty}} \\
& \quad \times \sum_{j=0}^{\infty} \frac{\left(\mu, q \sqrt{\mu},-q \sqrt{\mu}, a b q / d, a b q / c, a^{2} q / c d, a b q / c d ; q^{2}\right)_{j}}{\left(q^{2}, \sqrt{\mu},-\sqrt{\mu}, a^{2} q^{3} / c^{2} d, a^{2} q^{3} / c d^{2}, a b q^{3} / c d, a^{2} q^{3} / c d ; q^{2}\right)_{j}} \\
& \quad \times\left(-\frac{a q^{4}}{b c d}\right)^{j} a^{2\left(\begin{array}{l}
j \\
2
\end{array}\right)} .
\end{aligned}
$$

Now observe that by letting $g \rightarrow \infty$ in Watson's transformation formula [21, (3.4.1.5)] we get

$$
\begin{aligned}
& \sum_{j=0}^{\infty} \frac{(a, q \sqrt{a},-q \sqrt{a}, c, d, e, f ; q)_{j}}{(q, \sqrt{a},-\sqrt{a}, a q / c, a q / d, a q / e, a q / f ; q)_{j}}\left(-\frac{a^{2} q^{2}}{c d e f}\right)^{j} q^{\left(\begin{array}{l}
j \\
2
\end{array}\right)} \\
& \quad=\frac{(a q, a q / e f ; q)_{\infty}}{(a q / e, a q / f ; q)_{\infty}} \phi_{2}\left[\begin{array}{l}
a q / c d, e, f \\
a q / c, a q / d
\end{array} ; q, \frac{a q}{e f}\right] .
\end{aligned}
$$

Hence, the sum over $j$ in (5.13) equals

$$
\frac{\left(q^{2}, a^{3} b q^{4} / c^{2} d^{2} ; q^{2}\right)_{\infty}}{\left(a b q^{3} / c d, a^{2} q^{3} / c d ; q^{2}\right)_{\infty}} \phi_{3}\left[\begin{array}{c}
a b q / c d, a^{2} q / c d, a q^{2} / b c d \\
a^{2} q^{3} / c d^{2}, a^{2} q^{3} / c^{2} d
\end{array} ; q^{2}, q^{2}\right]
$$


and it follows from $(5.12)$ that

$$
\begin{aligned}
\sum_{k=0}^{\infty} & \frac{1-a q^{3 k}}{1-a} \frac{(a, b, q / b ; q)_{k}\left(c, d, a^{2} q / c d ; q^{2}\right)_{k}}{\left(q^{2}, a q^{2} / b, a b q ; q^{2}\right)_{k}(a q / c, a q / d, c d / a ; q)_{k}} q^{k} \\
= & \frac{(a q, a q / c d ; q)_{\infty}\left(a b q / c, a b q / d, a q^{2} / b c, a q^{2} / b d ; q^{2}\right)_{\infty}}{(a q / c, a q / d ; q)_{\infty}\left(a b q, a b q / c d, a q^{2} / b, a q^{2} / b c d ; q^{2}\right)_{\infty}} \\
& +\frac{a q(a q, b, q / b ; q)_{\infty}\left(c, d, a^{2} q^{3} / c d^{2}, a^{2} q^{3} / c^{2} d ; q^{2}\right)_{\infty}}{c d(a q / c, a q / d, c d / a ; q)_{\infty}\left(a b q, a b q / c d, a q^{2} / b, a q^{2} / b c d ; q^{2}\right)_{\infty}} \\
& \times{ }_{3} \phi_{2}\left[\begin{array}{c}
a b q / c d, a^{2} q / c d, a q^{2} / b c d \\
a^{2} q^{3} / c d^{2}, a^{2} q^{3} / c^{2} d
\end{array} q^{2}, q^{2}\right] .
\end{aligned}
$$

Hence, replacing $a, b, c, d$, respectively, by $a b c q, d, b c q$, $a c q$, we obtain the quadratic summation formula

$$
\begin{aligned}
\sum_{k=0}^{\infty} & \frac{1}{-a b c q^{3 k+1}} \frac{(a b c q, d, q / d ; q)_{k}\left(b c q, a c q, a b q ; q^{2}\right)_{k}}{1-a b c q} q^{k} \\
& -\frac{\left(a b c q^{2}, d, q b c q^{3} / d, a b c d q^{2} ; q^{2}\right)_{k}(a q, b q, c q ; q)_{k}}{c(a q, b q, c q ; q)_{\infty}\left(d / c, q / c d, a b c d q^{2}, a b c q^{3} / d ; q^{2}\right)_{\infty}} \\
& \times{ }_{3} \phi_{2}\left[\begin{array}{c}
a b q, d / c, q / c d \\
a q^{2} / c, b q^{2} / c
\end{array} ; q^{2}, q^{2}\right] \\
= & \frac{\left(a b c q^{2}, 1 / c ; q\right)_{\infty}\left(a d q, b d q, a q^{2} / d, b q^{2} / d ; q^{2}\right)_{\infty}}{(a q, b q ; q)_{\infty}\left(d / c, q / c d, a b c d q^{2}, a b c q^{3} / d ; q^{2}\right)_{\infty}}
\end{aligned}
$$

Note that the above ${ }_{3} \phi_{2}$ series is balanced (Saalschützian) and hence summable when it terminates. Formula (5.2) follows from (5.16) and the Sears' [20, (10.1)] transformation formula

$$
\left.{ }_{3} \phi_{2}\left[\begin{array}{c}
a, b, c \\
e, f
\end{array} ; q, \frac{e f}{a b c}\right]=\frac{\left(\frac{f}{c}, \frac{e f}{a b} ; q\right)_{\infty}{ }_{3} \phi_{2}\left[\begin{array}{c}
\frac{e}{a}, \frac{e}{b}, c \\
e, \frac{e f}{a b}
\end{array} ; q, \frac{f}{c}\right.}{\left(f, \frac{e f}{a b c} ; q\right)_{\infty}}\right]
$$

In a letter to the author, Mizan Rahman gave a longer proof of (5.2) in which he started with formula (1.10). He also showed that the right side of (5.2) could be written as a sum of an ${ }_{8} \phi_{7}$ series and a ${ }_{4} \phi_{3}$ series which is symmetric in $a, b, c$. His proofs in [19] of other nonterminating quadratic summation formulas can be shortened by using (1.14) in place of (1.10).

By starting with the special case

$$
\sum_{k=0}^{n} \frac{\left(1-a q^{4 k}\right)\left(1-b q^{-2 k}\right)}{(1-a)(1-b)} \frac{(a, b ; q)_{k}\left(q^{-3 n}, a q^{3 n} / b ; q^{3}\right)_{k}}{\left(q^{3}, a q^{3} / b ; q^{3}\right)_{k}\left(a q^{1+3 n}, b q^{1-3 n} ; q\right)_{k}} q^{3 k}=\delta_{n, 0}
$$


of (1.14) and setting

$$
B_{n}=\frac{\left(q^{3} \sqrt{a / b},-q^{3} \sqrt{a / b}, c, a^{2} b / c ; q^{3}\right)_{n}}{\left(\sqrt{a / b},-\sqrt{a / b}, a q^{3} / b c, c q^{3} / a b^{2} ; q^{3}\right)_{n}} q^{3 n}
$$

we get

$$
\begin{aligned}
1= & \sum_{n=0}^{\infty} \frac{\left(a / b ; q^{3}\right)_{n}(1 / b ; q)_{3 n}}{\left(q^{3} ; q^{3}\right)_{n}(a q ; q)_{3 n}} B_{n} \delta_{n, 0} \\
= & \sum_{k=0}^{\infty} \frac{1-a q^{4 k}}{1-a} \frac{(a, b ; q)_{k}\left(a / b ; q^{3}\right)_{2 k}(q / b ; q)_{2 k}}{\left(q^{3}, a q^{3} / b ; q^{3}\right)_{k}(a q ; q)_{4 k}} \\
& \times \frac{\left(q^{3} \sqrt{a / b},-q^{3} \sqrt{a / b}, c, a^{2} b / c ; q^{3}\right)_{k}}{\left(\sqrt{a / b},-\sqrt{a / b}, a q^{3} / b c, c q^{3} / a b^{2} ; q^{3}\right)_{k}} b^{-k} q^{3 k+2\left(\begin{array}{l}
k \\
2
\end{array}\right)} \\
& \times{ }_{8} W_{7}\left(a q^{6 k} / b ; a^{2} b q^{3 k} / c, c q^{3 k}, q^{2 k} / b, q^{2 k+1} / b, q^{2 k+2} / b ; q^{3}, q^{3}\right) .
\end{aligned}
$$

Now apply (5.11), with $b$ replaced by $a^{2} b q^{3 k} / c$, to the above ${ }_{8} W_{7}$, proceed as in the derivation of (5.13), and use the following $c \rightarrow \infty$ limit case of (5.14)

$$
\begin{aligned}
& \sum_{j=0}^{\infty} \frac{(a, q \sqrt{a},-q \sqrt{a}, d, e, f ; q)_{j}}{(q, \sqrt{a},-\sqrt{a}, a q / d, a q / e, a q / f ; q)_{j}}\left(\frac{a^{2} q^{2}}{d e f}\right)^{j} q^{2\left(\begin{array}{l}
j \\
2
\end{array}\right)} \\
& \quad=\frac{(a q, a q / e f ; q)_{\infty}}{(a q / e, a q / f ; q)_{\infty}}{ }_{2} \phi_{1}\left[\begin{array}{l}
e, f \\
a q / d
\end{array} ; q, \frac{a q}{e f}\right]
\end{aligned}
$$

to obtain the cubic summation formula

$$
\begin{aligned}
\sum_{k=0}^{\infty} & \frac{1}{-a q^{4 k}} \frac{(a, b ; q)_{k}\left(q / b, q^{2} / b ; q^{2}\right)_{k}\left(c, a^{2} b / c ; q^{3}\right)_{k}}{1-a} q^{k} \\
& +\frac{a b^{2}}{c} \frac{(a q, b q, 1 / b ; q)_{\infty}\left(c, a^{2} b q^{3} / c^{2} ; q^{3}\right)_{\infty}}{(a b, c q / a b, a q / c ; q)_{\infty}\left(a b^{2} / c, a q^{3} / b ; q^{3}\right)_{\infty}} \\
& \times{ }_{2} \phi_{1}\left[\begin{array}{c}
a b^{2} / c, a^{2} b / c \\
a^{2} b q^{3} / c^{2}
\end{array} q^{3}, q^{3}\right] \\
= & \frac{(a q, a b / c ; q)_{\infty}\left(a b^{2}, a q^{3} / b c ; q^{3}\right)_{\infty}}{(a b, a q / c ; q)_{\infty}\left(a b^{2} / c, a q^{3} / b ; q^{3}\right)_{\infty}}
\end{aligned}
$$

For some additional cubic summation formulas, see [19].

Notice that by replacing $a, b, c$ in (5.22) by $q^{3 a}, q^{3 b}, q^{3 c}$, letting $q \rightarrow 1$, and using Gauss' formula [21, (III.3), p. 243] and the identity $\Gamma(a) \Gamma(1-a)=$ $\pi / \sin \pi a$, we obtain

$$
\begin{aligned}
{ }_{7} F_{6}\left[\begin{array}{c}
3 a, 1+3 a / 4,3 b,(1-3 b) / 2,1-3 b / 2, c, 2 a+b-c \\
3 a / 4,1+a-b,(1+3 a+3 b) / 2,3(a+b) / 2,1+3 a-3 c, 1+3 c-3 a-3 b
\end{array} ; 1\right] \\
=\frac{\Gamma(3 a+3 b) \Gamma(1+3 a-3 c) \Gamma(a+2 b-c) \Gamma(1+a-b)}{\Gamma(3 a+1) \Gamma(3 a+3 b-3 c) \Gamma(a+2 b) \Gamma(1+a-b-c)} \\
\quad \times\left\{1+\frac{\sin 3 \pi b \sin \pi c}{\sin 3 \pi(a+b-c) \sin \pi(a+2 b)}\right\} .
\end{aligned}
$$


Mizan Rahman pointed out in a letter to the author that a special case of this formula evaluates Gosper's ${ }_{7} F_{6}$ series in $[14,(6.1)]$ for the case $b=2 a$.

If, instead of starting with (5.6) or (5.18), we start with the special case

$$
\sum_{k=0}^{n} \frac{\left(1-a q^{5 k}\right)\left(1-b q^{-3 k}\right)}{(1-a)(1-b)} \frac{(a, b ; q)_{k}\left(q^{-4 n}, a q^{4 n} / b ; q^{4}\right)_{k}}{\left(q^{4}, a q^{4} / b ; q^{4}\right)_{k}\left(a q^{1+4 n}, b q^{1-4 n} ; q\right)_{k}} q^{4 k}=\delta_{n, 0}
$$

of $(1.14)$ and set

$$
C_{n}=\frac{\left(q^{4} \sqrt{a / b},-q^{4} \sqrt{a / b}, a^{2} b^{2} / q^{2} ; q^{4}\right)_{n}}{\left(\sqrt{a / b},-\sqrt{a / b}, q^{6} / a b^{3} ; q^{4}\right)_{n}} q^{4 n}
$$

we get

$$
\begin{aligned}
1= & \sum_{n=0}^{\infty} \frac{\left(a / b ; q^{4}\right)_{n}(1 / b ; q)_{4 n}}{\left(q^{4} ; q^{4}\right)_{n}(a q ; q)_{4 n}} C_{n} \delta_{n, 0} \\
= & \sum_{k=0}^{\infty} \frac{1-a q^{5 k}}{1-a} \frac{(a, b ; q)_{k}(q / b ; q)_{3 k}\left(a^{2} b^{2} / q^{2} ; q^{4}\right)_{k}\left(a q^{4} / b ; q^{4}\right)_{2 k}}{\left(q^{4}, a q^{4} / b, q^{6} / a b^{3} ; q^{4}\right)_{k}(a q ; q)_{5 k}} b^{-k} q^{4 k+3\left(\begin{array}{l}
k \\
2
\end{array}\right)} \\
& \quad \times{ }_{8} W_{7}\left(a q^{8 k} / b ; a^{2} b^{2} q^{4 k-2}, q^{3 k} / b, q^{3 k+1} / b, q^{3 k+2} / b, q^{3 k+3} / b ; q^{4}, q^{4}\right) .
\end{aligned}
$$

By applying (5.11), with $b$ replaced by $a^{2} b^{2} q^{4 k-2}$, to the above ${ }_{8} W_{7}$ and using the $f \rightarrow \infty$ limit case of $(5.21)$

$$
\begin{aligned}
& \sum_{j=0}^{\infty} \frac{(a, q \sqrt{a},-q \sqrt{a}, d, e ; q)_{j}}{(q, \sqrt{a},-\sqrt{a}, a q / d, a q / e ; q)_{j}}\left(-\frac{a^{2} q^{2}}{d e}\right)^{j} q^{3\left(\frac{j}{2}\right)} \\
& \quad=\frac{(a q ; q)_{\infty}}{(a q / e ; q)_{\infty}} \phi_{1}\left[\begin{array}{c}
e \\
a q / d
\end{array} ; q, \frac{a q}{\dot{e}}\right],
\end{aligned}
$$

we obtain the quartic summation formula

$$
\begin{aligned}
\sum_{k=0}^{\infty} & \frac{1}{-a q^{5 k}} \frac{(a, b ; q)_{k}\left(q / b, q^{2} / b, q^{3} / b ; q^{3}\right)_{k}\left(a^{2} b^{2} / q^{2} ; q^{4}\right)_{k}}{1-a} q^{k} \\
& +\frac{a b^{3}}{q^{2}} \frac{\left.\left(a b, a q^{4} / b ; q^{4}\right)_{k}\left(a b q, a b, a b / q ; q^{2}\right)_{k}\left(q^{3} / a b^{2} ; q\right)_{k} ; q\right)_{\infty}\left(a b / q ; q^{2}\right)_{\infty}\left(q^{4}, a b^{3} / q^{2}, a q^{4} / b ; q^{4}\right)_{\infty}}{(a b, 1 / b ; q)_{\infty}\left(a^{2} b^{2} q^{2} ; q^{4}\right)_{\infty}} \\
& \times{ }_{1} \phi_{1} \cdot\left[\begin{array}{l}
a^{2} b^{2} / q^{2} \\
\left.a^{2} b^{2} q^{2} ; q^{4}, a b^{3} q^{2}\right]
\end{array}\right. \\
= & \frac{\left(a q, a b^{2} / q^{2} ; q\right)_{\infty}}{(a b ; q)_{\infty}\left(a b / q ; q^{2}\right)_{\infty}\left(a q^{4} / b, a b^{3} / q^{2} ; q^{4}\right)_{\infty}} .
\end{aligned}
$$

A $q$-analogue of Gosper's strange evaluation $[14,(6.1)]$ and additional results will be given in joint paper with Mizan Rahman. 


\section{REFERENCES}

1. R. P. Agarwal and A. Verma, Generalized basic hypergeometric series with unconnected bases, Proc. Cambridge Phil. Soc. 63 (1967), 727-734.

2. _ Generalized basic hypergeometric series with unconnected bases. II, Quart. J. Math. Oxford 18 (1967), 181-192; Corrigenda, ibid. 21 (1970), 384.

3. W. A. Al-Salam and A. Verma, On quadratic transformations of basic series, SIAM J. Math. Anal. 15 (1984), 414-420.

4. G. E. Andrews, On basic hypergeometric series, mock theta functions, and partitions. I, Quart. J. Math. 17 (1966), 65-135.

5. R. Askey and G. Gasper, Inequalities for polynomials, The Bieberbach Conjecture, (A. Baernstein, D. Drasin, P. Duren, and A. Marden, eds.), Math. Surveys Monogr., no. 21, Amer. Math. Soc., Providence, R.I., 1986, pp. 7-32.

6. W. N. Bailey, Generalized hypergeometric series, Cambridge Univ. Press, Cambridge, 1935.

7. Louis de Branges, A proof of the Bieberbach conjecture, Acta Math. 154 (1985), 137-152.

8. D. M. Bressoud, The Bailey lattice: an introduction, Ramanujan Revisited, (G. E. Andrews et al., eds), Academic Press, New York, 1988, pp. 57-67.

9. L. Carlitz, Some inverse relations, Duke Math. J. 40 (1973), 803-901.

10. J. L. Fields and M. E. H. Ismail, Polynomial expansions, Math. Comp. 29 (1975), 894-902.

11. J. L. Fields and J. Wimp, Expansions of hypergeometric functions in hypergeometric functions, Math. Comp. 15 (1961), 390-395.

12. G. Gasper, A short proof of an inequality used by de Branges in his proof of the Bieberbach, Robertson and Milin conjectures, Complex Variables 7 (1986), 45-50.

13. G. Gasper and Mizan Rahman, Basic hypergeometric series, Cambridge Univ. Press, (to appear).

14. I. Gessel and D. Stanton, Strange evaluations of hypergeometric series, SIAM J. Math. Anal. 13 (1982), 295-308.

15. __ Applications of q-Lagrange inversion to basic hypergeometric series, Trans. Amer. Math. Soc. 277 (1983), 173-201.

16. __ Another family of q-Lagrange inversion formulas, Rocky Mountain J. Math. 16 (1986), 373-384.

17. F. H. Jackson, Transformations of q-series, Messenger of Math. 39 (1910), 145-153.

18. Y. L. Luke, The special functions and their applications. II, Academic Press, New York, 1969.

19. Mizan Rahman, Some quadratic and cubic summation formulas for basic hypergeometric series, (to appear).

20. D. B. Sears, On the transformation theory of basic hypergeometric functions, Proc. London Math. Soc. (2) 53 (1951), 158-180.

21. L. J. Slater, Generalized hypergeometric functions, Cambridge Univ. Press, Cambridge, 1966.

22. H. M. Srivastava, Certain q-polynomial expansions for functions of several variables, IMA J. Appl. Math. 30 (1983), 315-323.

23. _ Certain q-polynomial expansions for functions of several variables. II, IMA J. Appl. Math. 33 (1984), 205-209.

24. D. Stanton, Recent results for the $q$-Lagrange inversion formula, Ramanujan Revisited, (G. E. Andrews, et al., eds.), Academic Press, New York, 1988, pp. 525-536.

25. A. Verma, Some transformations of series with arbitrary terms, Instit. Lombardo (Rend. Sc.) A 106 (1972), 342-353.

Department of Mathematics, Northwestern University, Evanston, Illinois 60208 